\title{
Toward Simultaneous Assessment of In and $N$ in InGaAsN Alloys by Quantitative STEM-ADF Imaging
}

\author{
V. Grillo,* K.Muller**, F.Glas***, K. Volz****, A.Rosenauer** \\ * CNR NANO-S3 Via Campi 213/A, 41100 Modena \& IMEM CNR, Parco Area delle Scienze \\ 37/A,I-43010Parma,Italy \\ ** Institut für Festkörperphysik, Universität Bremen, Otto-Hahn-Allee 1, D-28359 Bremen, \\ Germany \\ *** Laboratoire de Photonique et de Nanostructures, CNRS, Route de Nozay, 91460 Marcoussis, \\ France \\ **** Materials Science Center and Faculty of Physics, Philipps University Marburg, Germany
}

Scanning transmission electron microscopy with an annular dark field electron has proved to be a powerful analytical tool on atomic scale. In particular the possibility to extract quantitative chemical information has been demonstrated by an accurate comparison with frozen phonon simulations [1]. In fact in the High Angle (HAADF) limit the intensity contrast with a known reference material can be used to extract an alloy composition.

A fundamental requisite to reproduce experimental intensity is that the static atomic disorder (SD) induced by the presence of species with different covalent/ionic radii in the alloy are accounted for. In the field of semiconductors the correction due to SD has been proved to be important for the InGaAs alloy [1] and especially for the diluted GaAsN system where SD related effects are the main responsible of the contrast with GaAs [2]. In particular SD effects in GaAsN disappears at very high angle for which the GaAs - GaAsN contrast almost vanishes.

In this contribution we aim to demonstrate that the above observations can be used to create a novel methodology to extract both In and $\mathrm{N}$ content in the quaternary InGaAsN alloy. The InGaAsN is widely studied for its optoelectronic applications [3].

We have studied a sample comprising a 4 period multiple quantum well of InGaAsN with nominal compositions of $\operatorname{In}[\mathrm{x}]=20 \%$ and $\mathrm{N}[\mathrm{y}]=2.2 \%$. The sample has been grown by MOVPE..

Experiments have been performed on a Titan operated at $300 \mathrm{Kev}$ and with a convergence of 8.8 mrad.

The technique that we propose is based on the comparison of low angle (LA) and high angle ADF experiments that permits to alternatively switch on and off the $\mathrm{N}$ effects while retaining the main In effect. To prove quantitatively this method the dependence on [x] and [y] of HAADF $(50<\theta<301$ mrad) and LAADF $(26<\theta<161 \mathrm{mrad})$ has been plotted in fig $1 \mathrm{a}$, b. The simulations refer to a thickness of $88 \mathrm{~nm}$ relevant for comparison with experiments. All intensities have been normalized to the GaAs level.

The simulation, based on parallel computing multislice [1], shows that HAADF intensity is practically insensitive to $\mathrm{N}$ content while $\mathrm{N}$ affects importantly the LAADF intensity in fig $1 \mathrm{~b}$.

Fig 2 a,b shows the experimental images at HAADF and LAADF conditions of one of the QWs, The images have been normalized to the GaAs level in the barriers. The effect of surface strain relaxation is visible as dark contrast on the two sides of the quantum well and is expected to give artifacts in the interface regions which are therefore excluded from quantitative evaluations [4]. 
Fig $2 \mathrm{c}$ is a false color image of the difference between fig a,b and can be considered as mainly related to $\mathrm{N}$ distribution.

We also performed a quantitative evaluation of the average In and $\mathrm{N}$ based the intensity in the central part of the well. The composition set that produce the best fit between simulations and experiments is $[\mathrm{x}]=19 \%$ and $[\mathrm{y}]=1 \%$. This result can be considered as an important progress toward quantitative compositional evaluation of quaternary alloys whereas more accurate description of LAADF scattering is necessary [5].

\section{References}

[1] V. Grillo et al. Phys. Rev. B 77 (2008) 054103.

[2] X.Wu et al., J. Phys.: Condens. Matter 20 (2008) 075215.

[3] P.J. Klar Progress in Solid State Chemistry 31 (2003) 301-349

[4] V.Grillo Ultramicroscopy,109 ( 2009), 1453.

[5] The computations in this work has been performed under the HPC-EUROPA2 project (project number: 228398) with the support of the European Commission - Capacities Area - Research Infrastructures.
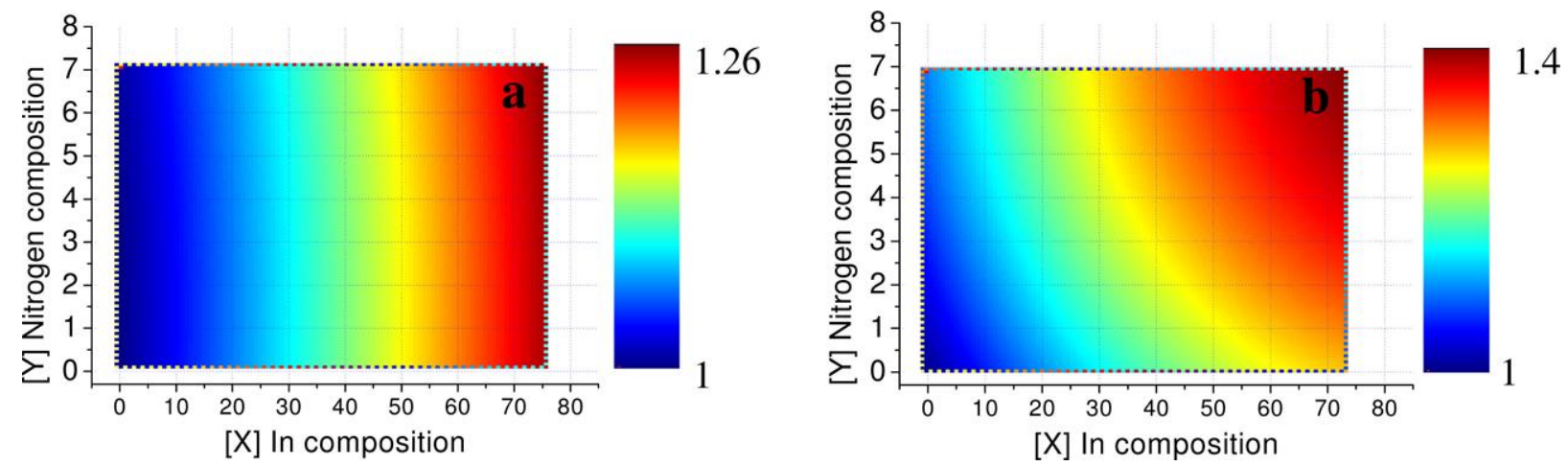

FIG. 1. Simulation of the dependence of the intensity, normalized to GaAs level, as a function of In $[\mathrm{x}]$ and $\mathrm{N}[\mathrm{y}]$ composition. Fig a,b refer to HAADF and LAADF imaging respectively (see text).
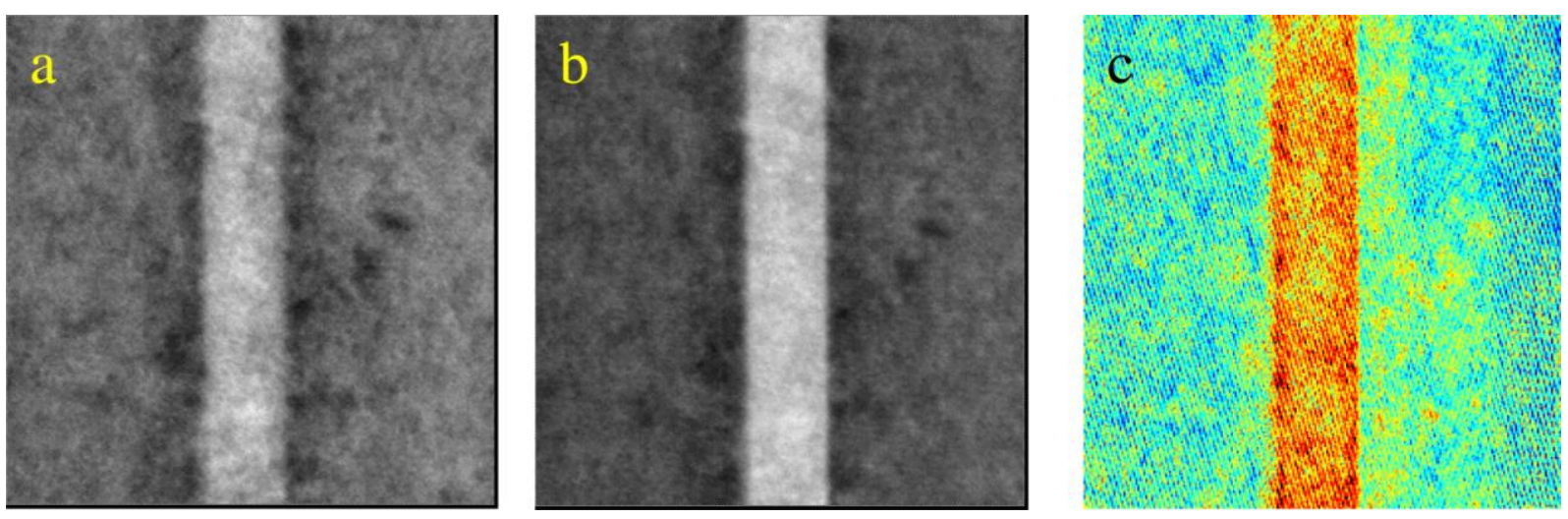

FIG. 2. Experimental image at HAADF (a) and LAADF (b) condition of one QW. Intensities are normalized to the GaAs barrier. False color image of the difference between image (b) and (a). 